\title{
Comunicado
}

[Communication $]$

\section{Florfenicol associado ou não ao cloprostenol no tratamento de retenção de placenta em vacas leiteiras}

[Florfenicol and sodium cloprostenol for the treatment of retained placenta in dairy cows]

\author{
M.P. Palhão ${ }^{1}$, A. Pereira ${ }^{2}$, C.A.C. Fernandes ${ }^{1,3^{*}}$, J. Rossi ${ }^{2}$, J.H.M. Viana ${ }^{1,4}$, M. Gioso ${ }^{1}$, \\ J.A.D. Garcia ${ }^{1}$, B.F.L. Alves ${ }^{3}$, E.R. Oliveira ${ }^{3}$ \\ ${ }^{1}$ Universidade José do Rosário Vellano - Unifenas - Alfenas, MG \\ ${ }^{2}$ Aluno de pós-graduação - Universidade José do Rosário Vellano - Unifenas - Alfenas, MG \\ ${ }^{3}$ Médico veterinário autônomo - Alfenas, MG \\ ${ }^{4}$ Embrapa Gado de Leite - Juiz de Fora, MG
}

A retenção de placenta (RP) é considerada patológica quando parte ou a totalidade dos anexos fetais não é liberada em até 12-16 horas após o parto (Kennedy, 1947; Erb et al., 1958; Callahan et al., 1971; Fernandes et al., 2001). Em rebanhos leiteiros, essa patologia pode acometer entre 10 e $15 \%$ dos animais, com maior incidência em vacas multíparas (24,4\%) (Kennedy, 1947; Erb et al., 1958; Callahan et al., 1971; Chew et al., 1977).

No período pós-parto imediato de animais que apresentam RP, instala-se uma infecção uterina puerperal (Fernandes et al., 2001). Este quadro pode evoluir para a retenção do conteúdo uterino, retardando o processo normal de involução uterina (Fernandes, 1999). A associação dessas duas alterações é responsável pela maior incidência de infecções uterinas aos 30 dias pósparto e pelo atraso no retorno à atividade reprodutiva (Freedman, 1994; Fernandes, 1999). Desta maneira, a eficiência dos tratamentos deve ser mensurada pela cura clínica e também pela fertilidade futura dos animais.

O presente estudo utilizou a combinação de um antibiótico e de um análogo da PFG $2 \alpha$ (cloprostenol) para testar as seguintes hipóteses: 1) uma única aplicação de florfenicol (dobro da dose intramuscular) pela via subcutânea poderia substituir o manejo da segunda dose administrada pela via intramuscular no

Recebido em 7 de março de 2013

Aceito em 30 de julho de 2013

*Autor para correspondência (corresponding author)

Email: carlos@biotran.com.br tratamento de RP em vacas leiteiras; 2) a associação entre terapia antibiótica e cloprostenol acelera o processo de involução uterina, reduz a incidência de infecção uterina pós-puerperal e melhora os índices reprodutivos de animais com RP pós-parto.

O estudo incluiu vacas das raças Holandesa e mestiças (Holandês/zebu) mantidas em sistema de semiconfinamento. O período experimental foi de 15 meses, e os animais diagnosticados com RP - não liberação da totalidade dos anexos fetais em até 16 horas após a expulsão do feto (Fernandes et al., 2001) - foram aleatoriamente distribuídos em um dos quatro grupos experimentais (Tab. 1). O delineamento utilizado foi inteiramente ao acaso (DIC), montado em arranjo fatorial $2 \times 2$, com dois níveis de dois fatores: 1) administração do florfenicol em duas formas distintas: intramuscular (IM) - 40mg para cada $15 \mathrm{~kg}$ de peso vivo, divididos em duas aplicações (20 horas após o parto e 48 horas após a primeira administração); ou subcutâneo (SC) $40 \mathrm{mg} / 15 \mathrm{~kg}$ em uma aplicação (20 horas após o parto); 2) inclusão ou não de cloprostenol, administrado em duas doses $(0,530 \mathrm{mg} / \mathrm{dose})$ às $20 \mathrm{~h}$ e às $48 \mathrm{~h}$ após o parto.

Entre 25 e 40 dias pós-parto (dpp), os animais foram examinados pela palpação retal, vaginoscopia e ultrassonografia (Scanner Falco Esaote). Por meio da vaginoscopia, foi avaliada a 
presença de secreções de origem uterina, e o aspecto foi classificado de acordo com o proposto por Sheldon e Dobson (2004), com escore variando de 0 (muco limpo ou translúcido) a 3 (pus liquefeito). A variável involução uterina foi mensurada em uma escala de 1 a 5 , com o escore 1 representando o útero totalmente na cavidade abdominal e 0 indicando quando mais da metade da massa uterina se encontrava na pelve (Fernandes et al., 2002). A avaliação da atividade ovariana consistiu na detecção ou não do corpo lúteo pela ultrassonografia transretal, conforme técnica descrita por Viana et al. (2000). Todos os procedimentos experimentais que envolvem animais foram aprovados pela Comissão de Ética no Uso de Animais (Ceua - Unifenas, Campus Alfenas, MG, Parecer n $\left.{ }^{\circ} 12 \mathrm{~A} / 2009\right)$.

Tabela 1. Descrição dos tratamentos dos animais com retenção de placenta

\begin{tabular}{|c|c|c|}
\hline Grupo & $\mathrm{N}$ & Tratamentos \\
\hline $1 *$ & 29 & Duas aplicações de florfenicol $^{1}(20 \mathrm{mg} / 15 \mathrm{~kg}$ PV) IM \\
\hline $2 *$ & 30 & Duas aplicações de florfenicol $(20 \mathrm{mg} / 15 \mathrm{~kg}) \mathrm{IM}+$ cloprostenol $^{2}$ \\
\hline $3 * *$ & 31 & Florfenicol $(40 \mathrm{mg} / 15 \mathrm{~kg}) \mathrm{SC}$ \\
\hline $4 * *$ & 30 & Florfenicol $(40 \mathrm{mg} / 15 \mathrm{~kg}) \mathrm{SC}+$ cloprostenol \\
\hline Total & 120 & \\
\hline
\end{tabular}

* Florfenicol (IM - intramuscular) dois tratamentos: 20h e 48h após o parto.

** Florfenicol (SC - subcutâneo) apenas um tratamento 20 horas após o parto.

${ }^{1}$ Nuflor $^{\circledR}$ (MSD Saúde Animal, São Paulo, Brasil).

${ }^{2}$ 0,530mg de cloprostenol (Ciosin ${ }^{\circledR}$ - MSD Saúde Animal, São Paulo, Brasil) administrado às 20h e às 48h após o parto.

O histórico reprodutivo dos animais foi posteriormente avaliado, e os seguintes indicadores de desempenho reprodutivo calculados: 1) intervalo do parto à primeira inseminação (P/IA); 2) intervalo do parto à concepção $(\mathrm{P} / \mathrm{C})$ e 3$)$ número de serviços por concepção (S/C).

$\mathrm{Na}$ análise estatística, as variáveis discretas infecção uterina, atividade ovariana e número de serviços por concepção - foram submetidas à estatística de Kruskal-Wallis para se compararem os quatro grupos. Para a comparação de dois grupos (tratados ou não com cloprostenol), utilizou-se a estatística de Wilcoxon; em ambos os casos, o teste de qui-quadrado foi utilizado para encontrar diferenças estatísticas. Os dados de P/C foram submetidos ao teste de Komolgorov-Smirnov, e transformações em logaritmo e raiz quadrada foram processadas quando os dados não respondiam aos princípios de distribuição normal. Desta forma, os dados referentes ao intervalo P/IA foram transformados em logaritmo e aqueles referentes ao intervalo $\mathrm{P} / \mathrm{C}$ foram transformados em raiz quadrada. Diferenças entre as médias dos grupos tratados ou não com cloprostenol foram acessadas pelo teste $t$ de Student. Adicionalmente, os intervalos do parto à primeira inseminação e do parto à concepção foram submetidos à distribuição de
Kaplan-Meier, e gráficos de sobrevivência foram plotados ao longo dos dias pós-parto. A estatística Log-Rank sugere que as curvas diferem quanto à velocidade com que os animais foram inseminados ou conceberam ao longo dos $\mathrm{dpp}(\mathrm{s})$. Probabilidades menores que $5 \%$ foram consideradas significativas, e aquelas $>5$ e $\leq 10$ foram consideradas aproximações ou tendências. Todas as análises foram realizadas no programa estatístico SAEG (Ribeiro Júnior, 2001).

Durante o período experimental, foram avaliados 579 partos, dos quais 120 vacas apresentaram a retenção dos anexos fetais, demonstrando uma ocorrência de $20,7 \%$ nos rebanhos estudados.

O efeito da interação não foi significativo para nenhuma das variáveis analisadas, de forma que os fatores foram estudados separadamente. O modo de administração do florfenicol - 40mg em dose única (SC) ou dividida (IM) - não afetou $(\mathrm{P}>0,05)$ o processo de involução ou a distribuição dos escores de infecção uterina. Porém, os animais tratados com o antibiótico se concentraram nos melhores escores de involução (4 e 5 - 65,8\%) e infecção (0 e 1 - 69,2\%), independentemente do modo de administração.

A significância encontrada na estatística de Kruskal-Wallis ( $\mathrm{P}<0,001 ;$ Tab. 2) deve-se à 
inclusão do análogo da prostaglandina ao tratamento antibiótico. Os animais não tratados com cloprostenol apresentaram uma distribuição uniforme nos escores de involução uterina (Tab.
2). De outra forma, os animais tratados com cloprostenol se concentraram nos escores 4 $(24 / 60)$ e $5(25 / 60)$.

Tabela 2. Distribuição dos escores de involução uterina nos grupos tratados ou não com cloprostenol ${ }^{1}$

\begin{tabular}{lcccccc}
\multirow{2}{*}{ Grupo } & \multicolumn{5}{c}{ Escore de involução uterina ${ }^{2}$} & \multirow{2}{*}{ Total } \\
\cline { 2 - 5 } & 1 & 2 & 3 & 4 & 5 & 60 \\
\hline Sem cloprostenol & $0(0,0)$ & $12(20,0)^{3}$ & $18(30,0)$ & $17(28,3)$ & $13(21,7)$ & 60 \\
Cloprostenol & $0(0,0)$ & $0(0,0)$ & $11(18,3)$ & $24(40,0)$ & $25(41,7)$ & 60 \\
\hline Total & $0(0,0)$ & $12(10,0)$ & $29(24,2)$ & $41(34,2)$ & $38(31,7)$ & 120 \\
\hline
\end{tabular}

A administração de prostaglandina no período pós-parto de vacas com RP também aumentou $(\mathrm{P}<0,01)$ o percentual de animais no escore $0 \mathrm{de}$ infecção uterina (Fig. 1) e contribuiu para um maior percentual (aproximação $\mathrm{P}<0,1)$ de animais com CL entre 25 e $40 \mathrm{dpp}(55,0$ vs. $41,7 \%)$.

A curva de sobrevivência (Fig. 2A) aponta que os animais tratados com prostaglandina foram mais rapidamente inseminados $(\mathrm{P}<0,03)$ no período pós-parto do que aqueles não tratados. Esse fato refletiu em um intervalo mais curto $(\mathrm{P}<0,03)$ do parto à primeira inseminação (74,9 $\pm 18,5$ vs. 82,2 $\pm 22,0$ dias). O intervalo P/IA mais curto e o menor $(\mathrm{P}<0,05)$ número de serviços por concepção $(2,6 \pm 1,1$ vs. $3,0 \pm 1,3$ doses) contribuíram para uma redução acentuada ( $\mathrm{P}<0,001$; Fig. 2B) do número de animais não gestantes. Desta maneira, o intervalo do parto à concepção foi mais curto $(\mathrm{P}<0,03)$ nos animais tratados com cloprostenol $(126,2 \pm 45,2$ vs. $144,8 \pm 55,5$ dias).
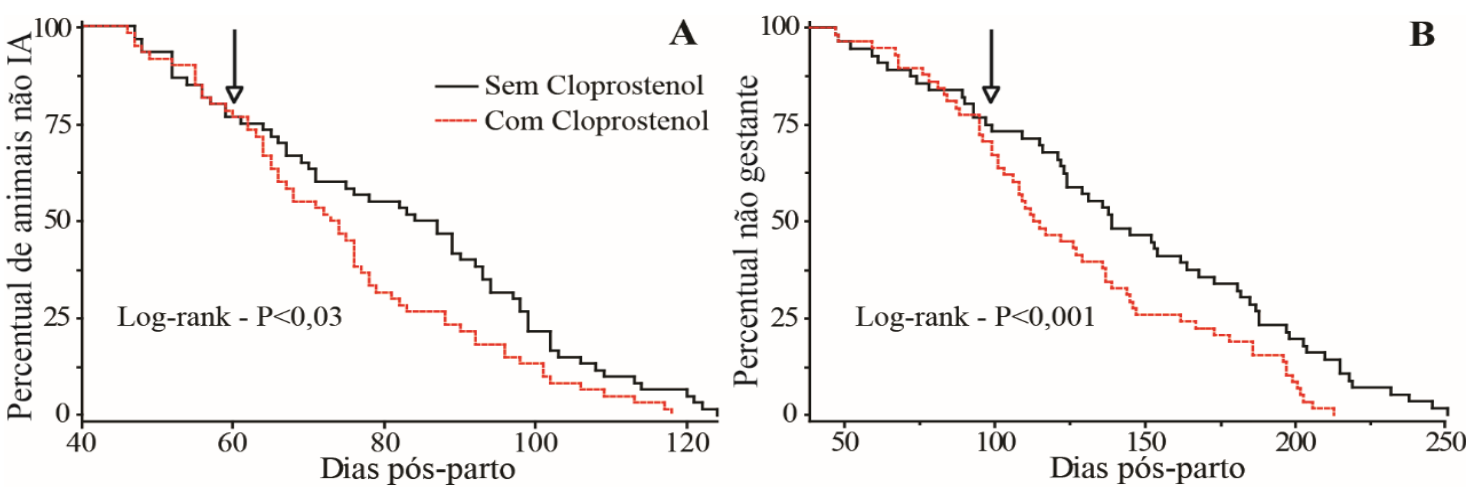

Figura 2. Gráfico de sobrevivência de Kaplan-Meier representando: A) o percentual de animais não inseminados e B) o percentual de animais não gestantes nos grupos sem ou com cloprostenol, ao longo dos dias pós-parto. As setas indicam o início da diferença entre os grupos. 
Um dos objetivos da realização deste estudo foi avaliar a substituição das duas doses IM do antibiótico por apenas uma dose pela via subcutânea. Sabe-se que o quadro clínico que se instala após a retenção de placenta coloca em risco a vida do animal, principalmente quando a RP é seguida por um quadro agudo de infecção uterina (Fernandes et al., 2001). Por isso há necessidade da terapia antibiótica, que visa reduzir a contaminação local e prevenir o acometimento sistêmico. O antibiótico utilizado (florfenicol), além de apresentar um amplo espectro de ação, tem eficiência comprovada no tratamento de infecções uterinas puerperais em bovinos (Fernandes, 1999). Considerando isso, o presente estudo avaliou somente alguns fatores relacionados ao retorno da atividade reprodutiva durante o período pós-parto. A via de administração e o número de aplicações do antibiótico não influenciaram a saúde uterina, avaliada pela palpação local e pelo exame vaginoscópico, bem como a atividade ovariana entre 25 e $40 \mathrm{dpp}$. Os índices reprodutivos também não foram influenciados pelos dois tratamentos antibióticos realizados neste estudo. Desta maneira, a primeira hipótese deste estudo foi comprovada e uma única dose de florfenicol (contendo 2x a dose IM), quando administrada pela via SC, pode substituir as duas aplicações pela via intramuscular no tratamento dos casos clínicos de RP em vacas leiteiras.

No presente estudo, a associação do análogo da PGF2 $\alpha$ ao antibiótico favoreceu os indicadores de desempenho reprodutivo dos animais tratados quando comparados àqueles que não receberam PGF2 $\alpha$. Estes fatos suportam a segunda hipótese deste estudo. A antecipação da atividade reprodutiva pós-parto (> percentual de animais com CL) foi o primeiro efeito da inclusão dessa droga ao tratamento e pode estar relacionada à maior rapidez no processo de involução uterina e à redução na ocorrência de infecções uterinas. Os mecanismos pelos quais a prostaglandina pode melhorar a saúde uterina pós-parto são controversos, mas o auxílio na expulsão do conteúdo contaminado e o aumento na imunidade uterina parecem ser ações comprovadas dessa molécula, quando administrada no pós-parto de vacas com infecção uterina (Lewis, 2004; Kasimanickam et al., 2005; Fernandes et al., 2007). As prostaglandinas e também seus análogos têm capacidade de estimular diretamente as células de defesa uterina, mesmo sem alterar as concentrações de progesterona (Lewis, 2004; Kasimanickam et al., 2005), situação que ocorre em vacas pós-parto, em que as concentrações desse esteroide são basais.

A melhora na saúde uterina e a antecipação no retorno à atividade reprodutiva no pós-parto parecem refletir no menor intervalo do parto à inseminação artificial. Mais especificamente a partir de $60 \mathrm{dpp}$, a velocidade com que os animais foram inseminados foi maior nos grupos tratados com cloprostenol. Esta observação, somada ao menor número de serviços por concepção, reduziu o número de vacas em aberto a partir dos 100 dpp. Outros estudos também demonstraram efeitos desse hormônio nos índices reprodutivos dos rebanhos (Fernandes et al., 2002; Weems et al., 2006), porém o presente artigo traz uma sequência ordenada de fatos que levaram a esta melhora no desempenho dos animais.

Nas condições experimentais estudadas, uma única aplicação via subcutânea de florfenicol se mostrou tão eficiente quanto o fracionamento desta em duas doses intramusculares, o que sugere uma redução do manejo durante o tratamento. Adicionalmente, observou-se que a associação de antibiótico e cloprostenol acelera a involução uterina, reduz a ocorrência de infecções, contribuindo para um melhor desempenho reprodutivo de vacas leiteiras com retenção de placenta.

Palavras-chave: eficiência reprodutiva, involução uterina, infecção uterina, período pós-parto

\begin{abstract}
The aim of this study was to compare the reproductive efficiency in dairy cows with retained placenta $(R P)$, treated with Florfenicol associated or not with Cloprostenol. One hundred and eight cows with RP from dairy herds in southern Minas Gerais state and northern São Paulo state, were included for this purpose. The experiment was designed in factorial 2X2. The four treatments encompassed two levels of each factor: 1) Florfenicol - 40mg/kg (IM) divided in two injections or 40mg $/ \mathrm{kg}$ (SC) in one injection
\end{abstract}


after calving; 2) Cloprostenol (0.530mg) - two injections associated with florfenicol treatment or without cloprostenol. The analysis of discrete variables used the Kruskal-Wallis (four groups) or Wilcoxon (two groups) statistic to compare groups. The Student " $T$ " test was used to compare means of continuous variables. The overall occurrence of RP was 20.7\%. The florfenicol groups had no effects on postpartum uterine health or subsequent reproductive efficiency of the cows. The cloprostenol injections of $R P$ hastened the uterine involution and decreased the occurrence of uterine infection, and increased the percentage of animals with corpus luteum between 25 and 40 days after parturition. The intervals from parturition to first artificial insemination (AI) and to conception, as well as the number of AI per conception were reduced in the cloprostenol treatment.

Keywords: reproductive efficiency, uterine involution, uterine infection, postpartum period

\section{AGRADECIMENTOS}

Os autores agradecem à Fundação de Amparo à Pesquisa do Estado de Minas Gerais (Fapemig) e ao Conselho Nacional de Pesquisa (CNPq), pelo suporte financeiro; à empresa Biotran Assessoria e Consultoria em Medicina Veterinária Ltda., pelo apoio financeiro e logístico para a realização do trabalho; à estudante de iniciação científica Mariana Elisabete de Oliveira Ferreira, pelo auxílio na formatação do manuscrito.

\section{REFERÊNCIAS}

CALLAHAN, C.J.; ERB, R.E.; SURVE, A.H. Variables influencing ovarian cycles in postpartum dairy cows. J. Anim. Sci., v.33, p.1053-1059, 1971.

CHEW, B.P.; KELLER, H.F.; ERB, R.E. et al. Periparturient concentrations of prolactin, progesterone and the estrogens in blood plasma of cows retaining and not retaining fetal membranes. J. Anim. Sci., v.44, p.1055-1060, 1977.

ERB, R.E.; HINZE, P.M.; GILDOW, E.M. et al. Retained fetal membranes- the effect on dairy cattle. J. Am. Vet. Med. Assoc., v.133, p.489496,1958.

FERNANDES, C.A.C. Alternativas para tratamento de retenção de placenta em gado de leite. Rev. Bras. Reprod. Anim., v.23, p.442-444, 1999.

FERNANDES, C.A.C.; COSTA, D.S.; VIANA, J.H.M. Impacto da retenção de placenta sobre a performance reprodutiva de vacas leiteiras. Rev. Bras. Rep. Anim., v.25, p.26-30, 2001.
FERNANDES, C.A.C.; VIEIRA, M.R.; VALLE, M.A.G. Efeito do cloprostenol sódico no pósparto de vacas leiteiras sobre a incidência de infecções uterinas e fertilidade futura. A Hora Vet., v.127, p.41-45, 2002.

FERNANDES, C.A.C.; FIGUEIREDO, A.C.S. Avanços na utilização de prostaglandinas, na reprodução de bovinos. Rev. Bras. Reprod. Anim., v.31, p.108-113, 2007.

FREEDMAN, S. Hormonal therapy in retained placenta and post parturient metritis. Israel J. Vet. Med., v.49, p.17-19, 1994.

KASIMANICKAM, R.; DUFFIELD, T.F.; FOSTER, C.J. et al. The effect of a single administration of cephafirin or cloprostenol on the reproductive performance of dairy cows with subclinical endometrits. Theriogenology, v.63, p.818-830, 2005.

KENNEDY, A. J. Retention of the placenta in the bovine. Vet. Rec., v.59, p.519-528, 1947.

LEWIS, G.S. Steroidal regulation of immune defenses. Anim. Reprod. Sci., v.82, p.281-294, 2004.

RIBEIRO JÚNIOR, J.I. Análises estatísticas no SAEG. Viçosa: Universidade Federal de Viçosa; 2001.

SHELDON, I.M.; DOBSON, H. Postpartum uterine health in cattle. Anim. Reprod. Sci., v.8283, p.295-306, 2004.

VIANA, J.H.M.; FERREIRA, A.M.; SA', W.F.; CAMARGO, L.S.A. Follicular dynamics in Zebu cattle. Pesq Agropec Br, v.35, p.250-2509, 2000.

WEEMS, C.W.; WEEMS, Y.S.; RANDEL, R.D. Prostaglandin and reproduction in female farm animals. Vet. J., v.171, p.206-228, 2006. 